\title{
Smart Traffic Light Signalling System using IR Sensors
}

\author{
*D. Lohitha ${ }^{1}$, Ch.SuneelKumar ${ }^{2}$, C.Muni kantha ${ }^{3}$ \\ ${ }^{1}$ Department of Electronics and communication Engineering, SVEW, SV colleges, Tirupati, A.P, India
}

\section{Received: 06th October 2017 Accepted: 14th November 2017, Published: 31st December 2017}

\begin{abstract}
:
Traffic light controlling becomes major issue with increase in automobiles and it also must to reduce time delays while going in traffic from long time delays at traffic signals. So, on considering this problem, we will go with Smart Traffic light signalling system using IR Sensors. This article explains how to reduce time delays at Higher traffic density areas. In this system, we are working with IR sensors to know about the traffic density at traffic signals. For this purpose we will arrange IR sensors at each road in Traffic junction. These IR sensors will find out the density of the traffic density at that road. These sensors are Interfaced to Microcontroller. With the detection of traffic density from IR sensors microcontroller will control the traffic signals accordingly on basis of density of traffic.
\end{abstract}

\section{Introduction}

At the Traffic junctions we are wasting too much of time due to long delay at traffic signalling. If we maintain this article as source we can rectify time delay at traffic delay on considering traffic density at particular road in traffic junction. Traffic density at road in a junction is measured with the IR sensor. If we consider four road junction, we have to use four IR sensor modules.

Initially we program our chosen microcontroller with normal time delay of some few seconds, if any road has more traffic density that road traffic signal will blink green LED to allow traffic to cross the junction until the road traffic density reaches to normal level. After getting into normal mode signals with blink as normal with some few seconds delay. All the operations we are doing with the help of microcontroller so, microcontroller is heart of our project. We have choosen ATMEGA32 microconroller for performing all these tasks easily. We are considering 3 LEDs (RED,GREEN,YELLOW) which will reflect our normal traffic signalling system. Glowing of each LED has performs with predefined time delays until if the road has high traffic density. Traffic LEDs are connected to Port B and D. IR sensors are connected to Port C. We are using push buttons to indicate the traffic density at the time of simulation to show. The logic levels of IR sensor are Logic 0 and Logic 1.If the Push button is pressed the logic level changes to 0 otherwise it will be logic 0 only. Logic 0 indicates traffic density is higher than normal, Logic 1 indicates traffic density is normal.

\section{Components used:}

Components what we require to perform our proposed project are:
a) ATMEGA32 Microcontroller
b) PCB Board
c) 12 LEDs
d) IR Sensor Modules
e) 12V Power supply
f) Serial cables
g) Connecting wires

\section{Block diagram:}

Block Diagram consists of 4 blocks Initially inputs are carried in by IR Sensor Module with the help of power supply, Microcontroller will process the information and sends output to LEDs to get the desired output of our proposed system.

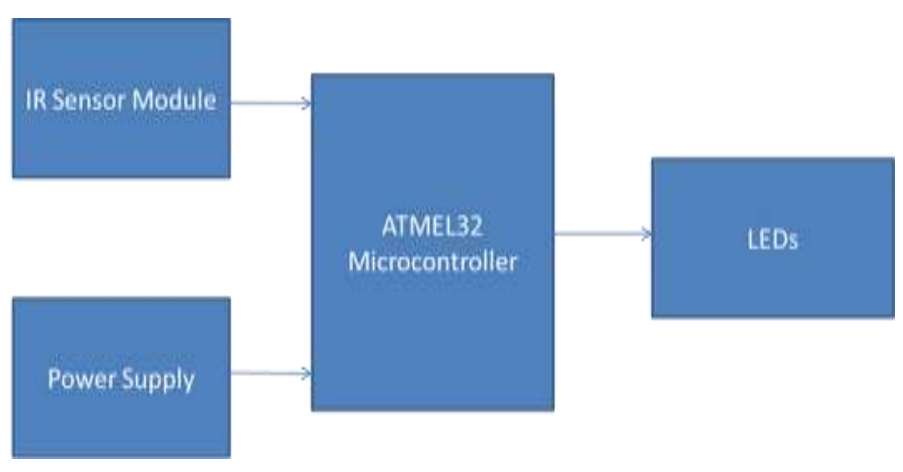

Fig 1: Block diagram of our proposed system.

\section{ATMEGA32 Microcontroller:}

ATMEGA32 Microcontroller have been used in our system. It is preferred because it consumes low power and is of CMOS type and it is 8-bit microcontroller possess enhanced RISC Architecture which implies we can execute powerful instructions in just a clock cycle.

\section{Features :}

The microcontroller features are as follows:

1. It exhibits high performance

2. It is of advanced RISC architecture with 131 instructions and execution in single cycle and have 32 general purpose registers of 8-bit. It has on-chip multiplier of 2-cycle.

3 . Highly executable memory segments which is nonvolatile flash program memory which is selfprogrammable and EEPROM of 1024 bytes and internal 
Helix Vol. 8(1): 2701- 2705

SRAM of 2GB, a programmable lock for system security purpose.

4.Two Timer/counter of 8-bit which has two separate modes. A Timer/counter of 16-bit.

5. It has PWM channels of count four.

6. A USART which is serially programmable, a serial USART port an Analog comparator which is onchip and an oscillator.

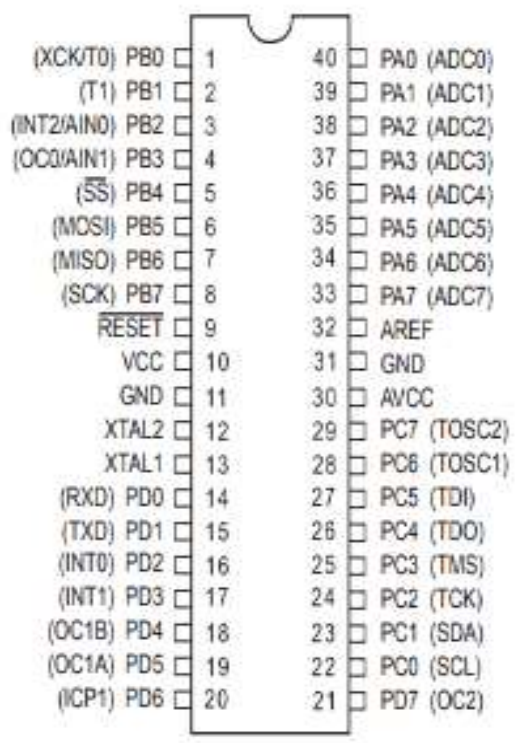

Fig 2: ATMEGA32 Microcontroller.

\section{LED:}

A light-emitting diode (LED) is mostly used component in many applications. It is made of material semiconductor which has two leads. It is also termed as $\mathrm{Pn}-$ junction diode which gets activated when light is exposed. The internal operation is when a sufficient voltage is supplied to the leads to the leads the electrons in the device will combine with the holes which in return results in the emission of photons in the form of energy. The LED colour is depended on the amount of energy emitted.

In place of Normal traffic signal lights we are using LEDs. We are using three coloured LEDs like RED, GREEN and YELLOW. Red Indicates to stop the vehicles. Green indicates to pass the vehicles. Yellow indicates ready to pass the vehicle through the junction. LEDs require greater than 3 Volts power supply to glow.
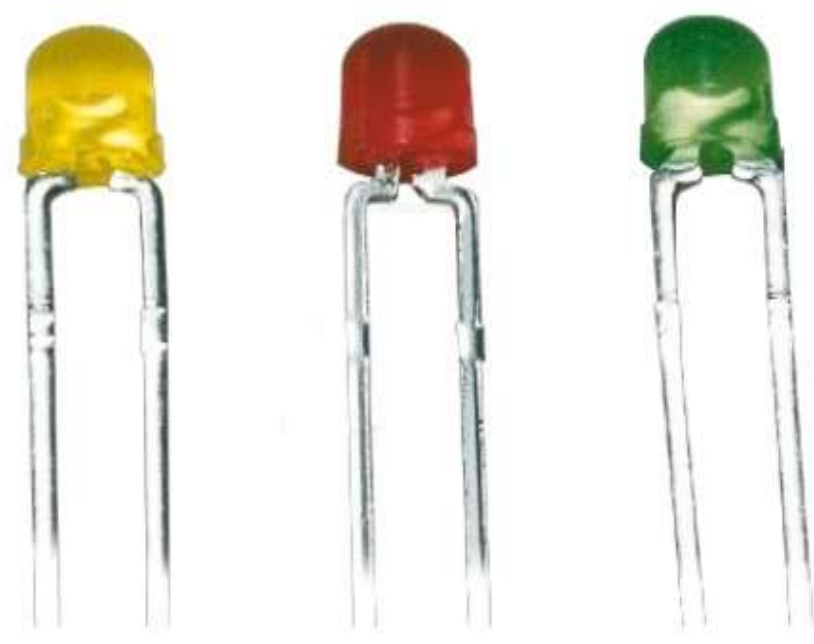

Fig 3: LEDs(Yellow,Red,Green).

\section{IR Sensor:}

IR sensor will emit IR rays which cannot be sensed by the humans but those are monitored with the help of camera. IR sensor has two LEDs ,one will act as transmitter another will act as receiver.

IR Sensor IR (INFRARED) sensor is based on LM 358 IC which is an Operational amplifier acting as comparator. The comparator compares the potentiometer analog voltages and the photodiode generated voltage. We are going to carry out comparision by applying those two voltages to the two terminals of IC and IC is going to generate digital output. The IR sensor is highly compatible that means it canbe combinated with many generally used microcontrollers. When photodiode generated voltage is greater than potentiometer analog voltage then the output of the system will be higher or else it will be lower.

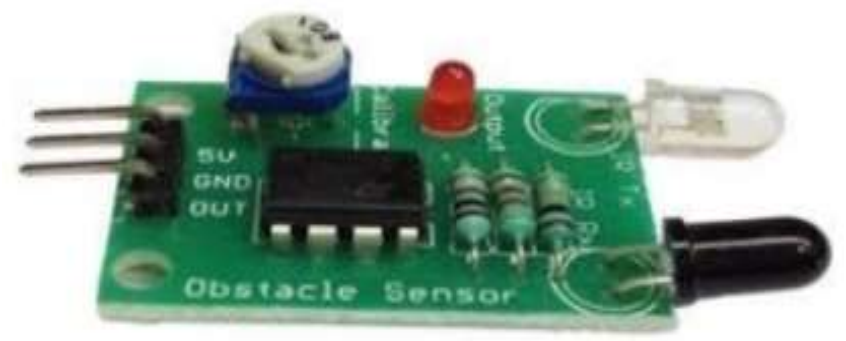

Fig 4: IR Sensor Module. 
Helix Vol. 8(1): 2701- 2705

\begin{tabular}{|c|c|c|}
\hline S.No & Pin & Description \\
\hline 1 & Pin1 & VCC \\
\hline 2 & Pin2 & Ground(GND) \\
\hline 3 & Pin3 & Output \\
\hline
\end{tabular}

Table 1: Pin Description of IR sensor Module IR Module consists of IR pair when we place any obstacle infront of it, IR receiver receives the IR rays transmitted by transmitter when the rays emitted by transmitter hits the obstqcle it will be received by the receiver. Those can be detected easily because it has resistance values of Mega

Ohms.

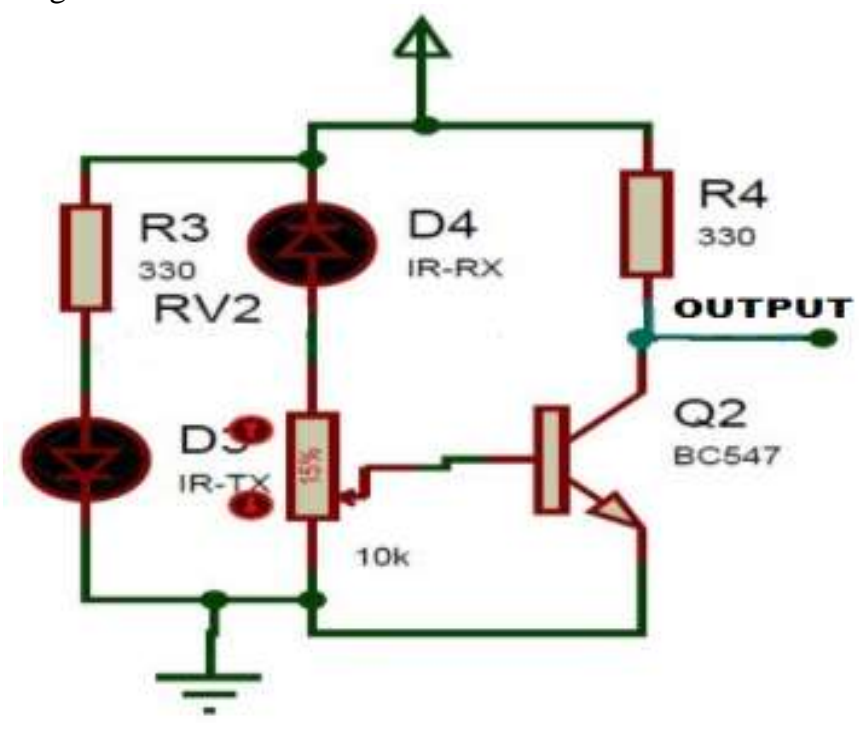

Fig 5: IR Sensor Module circuitry.

\section{Technical Specifications:}

1. The range is from $2-12 \mathrm{~cm}$.

2. In order to operate for maximum range a potentiometer is used.

3. We can find the difference between white and black that is nothing but line sensing.

4. For detecting Led indications are place on board.

5. Input voltage is $5 \mathrm{~V}$.

6. The output is compatible with TTL.

7. The IC is used as comparator by using ADC the values are

Converted to digital.

\section{Simulation:}

1. Proteus8.1 is a simulation software which is best suitable for various microcontroller designs.

2. Simple one which is easy to test program and signing of embedded electronics.

3. PROTEUS is a software which is not only used for simulating but also helps to combine components with 8051 microcontroller. Those components are like capacitance, LC's, LED's , Resistors, Inductors etc. A library is pre-determined so that once we have the lines of a module we have a scope to write the code defined for the application. Once the design is done then it is simulated with the simulator to get the output of the hardware components.

4. Now lets see how to design and simulate a project by using the PROTEUS software.

5. A name should be given for project and remaining details are kept same and proceed to click button.

6. The circuit diagram is framed by clicking the capture button of schematic and components are added by clicking the button $\mathrm{P}$ and then component button below design for selecting the components.

7. The components is chosen by typing the components name through the keyboard. After that select the components

8. The components is chosen by typing the component name through keyboard. After that select components and click the OK button.

9. Now make connections between the components to complete the circuit diagram.

Simulation of our proposed system consists of 12 LEDs ,4 IR Modules and 4 Push button switches which defines the density of traffic. with the clock wise manner the lights

will glow with time delay of few seconds.

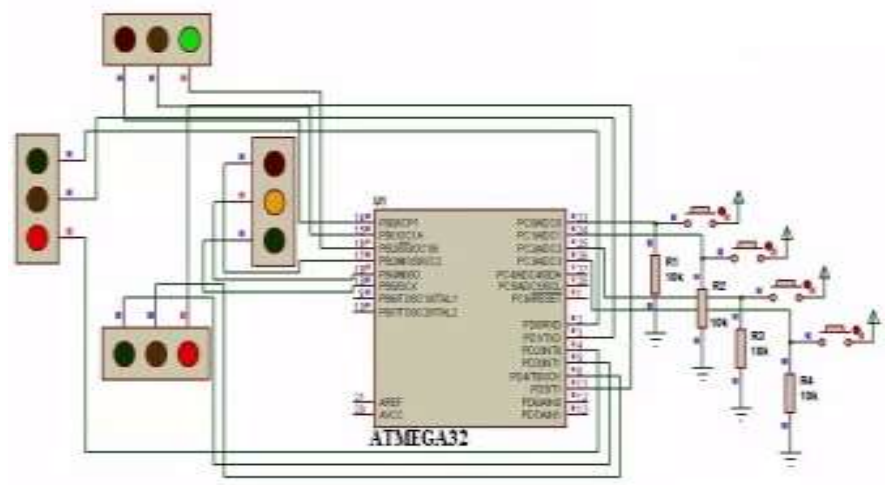

Fig 6: Simulation of Circuit with Normal traffic system 1.

In the above figure we can notice that among First set of LEDs glowing green LED which allows traffic to cross traffic junction from first road, among second set of LEDs glowing yellow LED from which indicates that ready to allow traffic to cross traffic junction from second road.

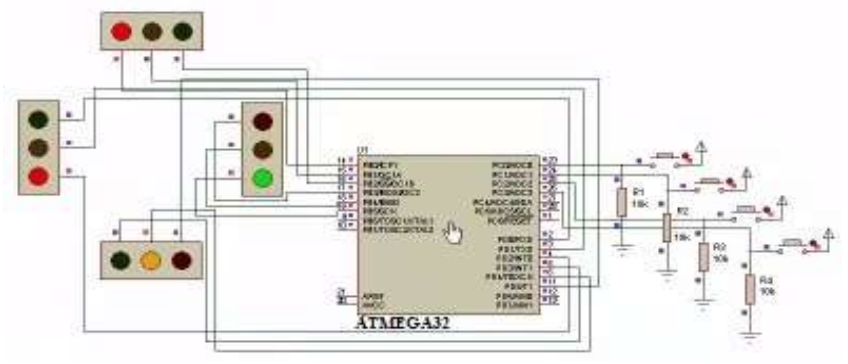


Fig 7: Simulation of Circuit with Normal traffic system 2. In the above figure we can notice that among Second set of LEDs glowing green LED which allows traffic to cross traffic junction from second road, among third set of LEDs glowing yellow LED from which indicates that ready to allow traffic to cross traffic junction from second road.

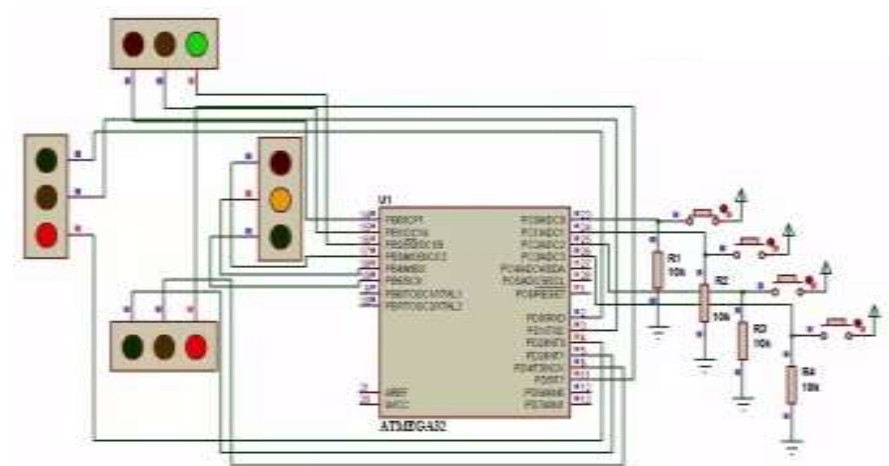

Fig 8: Simulation of Circuit with Smart IR Module 1 Detected Traffic Density.

In the above figure we can notice that among First set of LEDs glowing green LED irrespective of normal mode because of considering traffic density is high on pressing the push button1 which means IR sensor module1 detects traffic density allows traffic to cross traffic junction from road 1 until the traffic density becomes normal, then normal traffic based system on predefined delay of few seconds will continue.

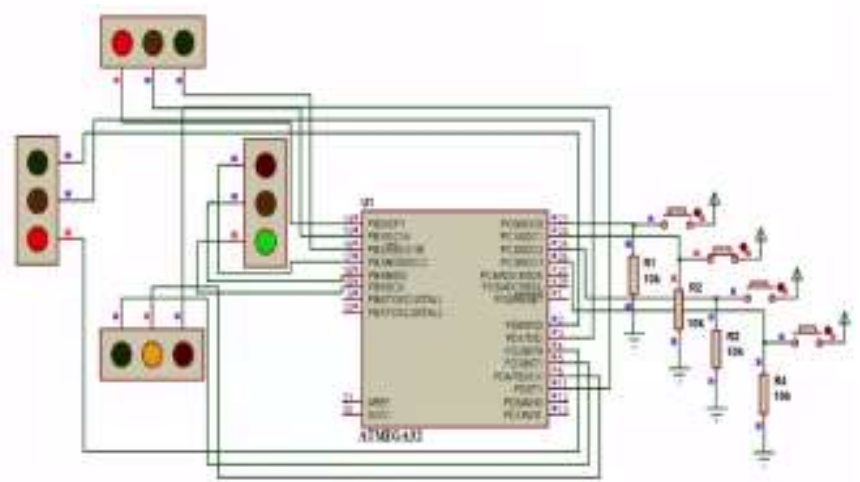

Fig 9: Simulation of Circuit with Smart IR Module 2 Detected Traffic Density.

In the above figure we can notice that among Second set of LEDs glowing green LED irrespective of normal mode because of considering traffic density is high on pressing the push button 2 which means IR sensor module2 detects traffic density allows traffic to cross traffic junction from road 2 until the traffic density becomes normal, then normal traffic based system on predefined delay of few seconds will continue.

\section{Results:}

Final output of our proposed system is achieved with full consideration of outputs what we expected to come with time basis. On placing some obstacle infront of IR Sensor module to identify that traffic density is high. Then green LED will glow to allow traffic in that particular path until traffic reaches to normal .Here we consider obstacle as traffic. After reaching traffic to normal state traffic signalling will glow with predefined time basis.



Fig 10: Final Output of our proposed system.

\section{Conclusion:}

In the project, the conventional traffic signalling system is replacing with system using IR sensors. Hardware are tested and the result is observed. Since the IR sensors costs less the project costs less. It can be implemented in various places in our day to day life in order to solve the conjestion problems in the cities where the number of vehicles are more. Presently in the general system usage the important vehicles like Ambulance, Fire Engines, Government vehicles etc those cannot be stopped for a longer duration.

So the traffic intensity should be cleared by just manipulating the normally timed signals whenever we have high density of traffic on one side then we should be given with green colour on that side. Once the density is cleared on all sides the general timing is followed which is pre defined in the program. The IR sensors are placed on the successive lengths on the roads. The IR receiver receives the signal once the Ir rays touches the obstacle.

\section{Future scope:}

As the system takes care of few of the drawbacks of the existing system, there exists a scope for the work expansion. The proposed system can be extended in order to clear the traffic for the emergency vehicles like fire engines, ambulances etc for those emergency vehicles even a second is also very valuable in that cases the traffic clearance plays a major role. By using the normal traffic system those vehicles also has to be follow the normal signal times which is not appropriate. So the traffic should be cleared at each and every point so the emerging 
Helix Vol. 8(1): 2701- 2705

vehicles moves on without any problems. In this project in future $\mathrm{i}$ can add module for sensing whose range is more than IR module. I will modify my coding for controlling the traffic signal according to density.

\section{References:}

1. Zhang Yuye \& Yan Weisheng, (2009) "Research of Traffic Signal Light Intelligent Control System Based On Microcontroller", First International Workshop on Education Technology and Computer Science,pp301303. Y.Zhao and Z.Ye, "A Low Cost GSM/GPRS Based Wireless Home Security System", IEEE Transactions on Consumer Electronics, Vol. 54, No. 2, pp.200-215, (2008).

2. Manoj Kanta Mainali \& Shingo Mabu (2010) "Evolutionary Approach for the Traffic Volume Estimation of Road Sections", pp100- 105, IEEE.

3. Shilpa S. Chavan, Dr. R. S. Deshpande \& J. G. Rana (2009) "Design of Intelligent Traffic Light Controller Using Embedded System" Second International Conference on Emerging Trends in Engineering and Technology, pp1086- 1091.

4. Xu Li, Wei Shu, Minglu Li, Hong-Yu Huang, Pei-En Luo, and Min-You Wu, "Performance Evaluation of Vehicle-Based Mobile Sensor Networks for Traffic Monitoring" IEEE 2009.

5. Manoj KantaMainali \& Shingo Mabu (2010) "Evolutionary Approach for the Traffic Volume Estimation of Road Sections", pp100- 105, IEEE.

6. Ahmed S. Salama, Bahaa K. Saleh, Mohamad M. Eassa "Intelligent Cross Road Traffic Management System", (ICRTMS), 2010 2nd International Conference on Computer Technology and Development (ICCTD 2010). 